\section{Forschende \\ Komplementärmedizin \\ Wissenschaft | Praxis | Perspektiven}

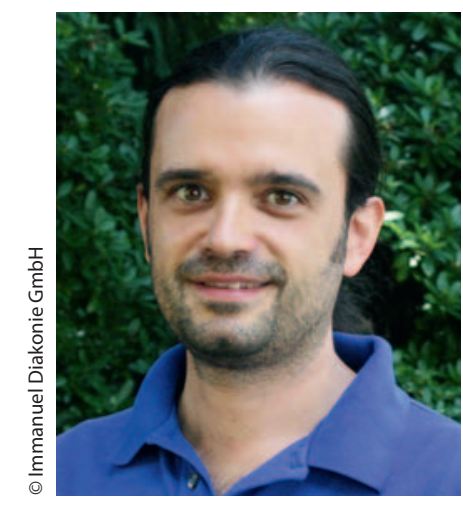

Christian S. Kessler

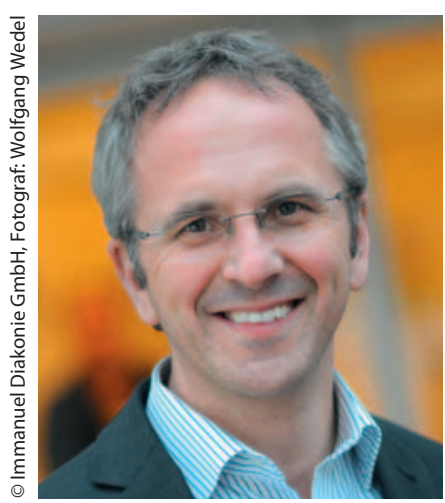

Andreas Michalsen

\section{Editorial}

Forsch Komplementmed 2016;23:64-65

DOI: $10.1159 / 000445519$
Published online: April 13, 2016

\title{
Veg* in der Medizin - schon längst viel mehr als nur ein Trend
}

\author{
Christian S. Kessler Andreas Michalsen \\ Abteilung für Naturheilkunde, Immanuel Krankenhaus und Charité - \\ Universitätsmedizin Berlin, Berlin, Deutschland
}

Sich vegetarisch zu ernähren, ganz zu schweigen davon, dies mit gesundheitlichen Vorteilen und therapeutischen Argumenten zu begründen, bedeutete noch vor 2 Jahrzehnten in Mitteleuropa in wechselnder Abfolge Vereinsamung am Tisch, mitleidige Blicke und Kommentare von Freunden und Familienangehörigen oder soziales Außenseitertum im öffentlichen kulinarischen Raum $[1,2]$. Die Situation hat sich seitdem in beeindruckender Geschwindigkeit verändert. Nicht nur, dass vegetarische Ernährungsweisen in der Bevölkerung seit Jahren kontinuierlich auf dem Vormarsch sind und das Stigma der Mangelernährung - zumindest in urbanen Ballungsräumen (nicht nur in Kosmopolen wie London oder Berlin) - längst verloren haben. Nein, mittlerweile gilt es in der einschlägigen Szene schon längst als «retro», wenn man von «vegetarisch» spricht; «vegan», «pflanzenbasiert» und «roh» sind längst die neuen diesbezüglichen gesellschaftlichen Trendbegriffe $[3,4]$. Trotz der großen Öffentlichkeit, die das Thema zunehmend genießt, sind zahlreiche Vorurteile (auf beiden Seiten) nur schwerlich aus der Welt zu schaffen. Daran scheinen auch die mittlerweile zahlreich vorliegenden Studien zu diesem Thema nichts zu ändern, obwohl sie zu einem guten Teil mit hochwertigen Studiendesigns, großen Probandenzahlen und Ergebnissen daherkommen, die nahelegen, dass ein Verzicht beziehungsweise eine deutliche Reduktion des Verzehrs von rotem Fleisch, Fleischprodukten und tierischen Proteinen viele gesundheitliche Benefits mit sich bringen [5-8] (von globalen ökologischen, ökonomischen und ethischen Aspekten ganz abgesehen). Kurioserweise ist dies vor allem in der Medizin und den Gesundheitswissenschaften hartnäckig der Fall, und inzwischen scheinen nicht selten Patienten und Patientinnen zu diesem Thema besser aufgeklärt als der ein oder andere Therapeut, der deshalb konsultiert wird. Dies hat sicher viel mit der Tatsache zu tun, dass das Thema Ernährung im Medizinstudium nach wie vor eine völlig untergeordnete Rolle spielt und es im Curriculum, auch von neuen Modell- und Reformstudiengängen, ein Außenseiterdasein fristet. In der öffentlichen Diskussion kommt komplizierend der Aspekt zum Tragen, dass es für Menschen in Wohlstandsregionen (insbesondere in Europa) schwierig zu sein scheint, sich von Traditionen und Gewohnheiten, die sich um tierische Produkte drehen, zu trennen bzw. darüber aus gesundheitlicher Sicht kritisch zu reflektieren. Bei solch emotionsgeladenen Themen hilft dann auch meist die beste Studie nichts, wenn diese nicht ins eigene paradigmatische Weltbild passt. Zu beobachten war dies überdeutlich bei der flutartigen und überwiegend emotionalen Pressereaktion auf die Publikation der WHO, mit der sie Fleisch und Fleischprodukte nun offiziell als krebserregend einstuft. Nicht zu vergessen: Deutschland (mit steigender Tendenz!) ist nach wie vor eines der am stärksten fleischproduzierenden und -exportierenden Länder [9] - eine starke Lobby trägt hier zweifellos maßgeblich dazu bei, eine sachliche Diskussion zum Thema effektiv zu vernebeln. Vergleiche zum Gebaren der Zigarettenindustrie in früheren Jahrzehnten sind schon an anderer Stelle mehrfach gezogen worden.

\section{KARGER}

Fax +497614520714
Prof. Dr. med. Andreas Michalsen

Abteilung für Naturheilkunde

Immanuel Krankenhaus und Charité - Universitätsmedizin Berlin

Königstraße 63, 14109 Berlin, Deutschland

a.michalsen@immanuel.de 
Dabei ist ein unübersehbarer Hang zum Extremismus und zur Polemik auf der anderen (Veggie-)Seite mitunter auch nicht besonders hilfreich; die moralinsaure Gutmenschendebatte ist dafür ein vortreffliches Beispiel.

Allerdings ist es spannend zu beobachten, wie sich die Fronten dennoch aufweichen. Jüngst sprach der Vorsitzende des Vegetarierbunds (VEBU) als Gastreferent auf dem Deutschen Fleischkongress, und bekannte Platzhirsche aus den deutschen Wurstregalen vertreiben seit neuestem schwerpunktmäßig pflanzenbasierte Wurst- und Fleischanaloga. Viele andere Hersteller folgen bis in die (Bio-)Tiefen der Discounter-Regale und erfreuen sich an 2-stelligen Erlöszuwächsen. Und an vielen Tischen, privaten wie öffentlichen, stellt sich zunehmend mehr Entspannung ein, eine polardichotome Atmosphäre weicht zunehmend einem toleranten Mitund/oder Nebeneinander. Ganz sicher der Fall ist dies in Berlin. Dort findet vom 22.-24. April zum 4. Mal VegMed statt, ein in Europa einzigartiger internationaler Kongress zu vegetarischer Ernährung in der Medizin. Die weltweit führenden Experten aus der Forschung zu vegetarischer Ernährung in der Medizin haben sich angekündigt. Einige davon werden in diesem Heft über ihre Forschungs- und Schwerpunktthemen berichten. Prof. Mark Messina liefert eine wesentliche Zusammenfassung der Datenlage zu Sojaprodukten bei Patientinnen mit Brustkrebs, ein vor allem auch aus hormoneller Sicht hochkontroverses Thema [10]. Prof. Claus Leitzmann, Nestor der deutschen Vegetarismusforschung und Vollwerternährung, schreibt über Eigenschaften und gesundheitliche Vorteile von sekundären Pflanzenstoffen [11]. Damit wird ein Thema behandelt, das bei der zunehmenden Diversifizierung in der wissenschaftlichen Vegetarierszene bisher völlig unterreprä- sentiert war und deshalb in dieser Form genau zum richtigen Zeitpunkt erscheint. Dr. Ram Manohar, einer der führenden Forscher aus der indischen Ayurvedamedizin und aus dem Land mit den mit Abstand meisten Vegetariern (40\%) kommend, liefert eine Synthese über Bedeutung und medizinischen Nutzen vegetarischer Ernährungsweise aus Sicht der traditionellen indischen Medizin [12]. Dr. Christian Kessler, Dr. Holger Cramer und Kollegen berichten über die Ergebnisse einer Online-Umfrage bezüglich Werten und Persönlichkeitsaspekten von Vegetariern [13]. Dr. Markus Keller und Stine Müller schreiben schließlich über das Thema, das nach wie vor ein großer und vielleicht auch der letzte Gegenstand ist, der immer noch zu starken Kontroversen und Widerständen in der Medizin führt - vegane Ernährung in Kindheit und Schwangerschaft [14]. Und einen der ganz großen Pioniere zum Thema, Prof. Gary Fraser aus Loma Linda, CA, USA, konnten wir für ein Gasteditorial gewinnen [15].

Wir hoffen darauf, dass diese Ausgabe der Forschenden KomplementärmEDIZIN einen entspannten, sachlichen und zukunftsweisenden Beitrag zu einem Thema leisten kann, das in unseren westlichen Gesellschaften immer mehr an Fahrt aufnimmt, dem aber bisher vielfach noch eine angemessene und wohltemperierte wissenschaftliche (und gesundheitspolitische) Begleitung fehlt. Letztlich ist das Thema Essen so essenziell, dass uns allen zu wünschen ist, unsere Ernährung zu nutzen, um mit uns selbst und anderen Menschen in gutem Kontakt zu sein und zu bleiben (und darüber hinaus perspektivisch vielleicht auch ein Stück mehr mit anderen Lebewesen). Unser Planet wird es uns wahrscheinlich danken [16].

\section{Literatur}

1 Leitzmann C: Vegetarian nutrition: past, present, future. Am J Clin Nutr 2014;100(Suppl 1):496S-502S.

2 Max-Rubner-Institut; Bundesforschungsinstitut für Ernährung und Lebensmittel (Hrsg): Nationale Verzehrsstudie II - Ergebnisbericht, Teil 1. Karlsruhe, 2008. www.bmel.de/SharedDocs/Downloads/Ernaehrung/ NVS_Ergebnisbericht.pdf?_blob=publicationFile (letzter Abruf 21. März 2016).

3 Ruby M B: Vegetarianism. A blossoming field of study. Appetite 2012;58:141-150.

4 Veser P, Taylor K, Singer S: Diet, authoritarianism, social dominance orientation, and predisposition to prejudice - results of a German survey. Br Food J 2015;117: 1949-1960.

5 Orlich MJ, Singh PN, Sabaté J, et al: Vegetarian dietary patterns and mortality in Adventist Health Study 2. JAMA Intern Med 2013;173:1230-1238.
6 Baron RB: Should we all be vegetarians? JAMA Intern Med 2013;173:1238-1239.

7 Michaëlsson K, Wolk A, Langenskiöld S, et al: Milk intake and risk of mortality and fractures in women and men: cohort studies. BMJ 2014;349:g6015.

8 Appleby PN, Thorogood M, Mann JI, Key TJ: The Oxford Vegetarian Study: an overview. Am J Clin Nutr 1999;70(3 Suppl):525S-531S.

9 Heinrich-Böll-Stiftung und ihre Landesstiftungen in Zusammenarbeit mit dem BUND (Hrsg): Fleischatlas 2016 - Deutschland Regional. Berlin, Heinrich-BöllStiftung, 2016.

10 Messina M: Impact of soy foods on the development of breast cancer and the prognosis of breast cancer patients. Forsch Komplementmed 2016;23:75-80.

11 Leitzmann C: Characteristics and health benefits of phytochemicals. Forsch Komplementmed 2016;23:6974.
Manohar R, Kessler CS: Āyurveda's contributions to vegetarian nutrition in medicine. Forsch Komplementmed 2016;23:89-94.

13 Kessler CS, Holler S, Zösch S, et al: Personality profiles, values and empathy: differences between lacto-ovovegetarians and vegans. Forsch Komplementmed 2016; 23:95-102.

14 Keller M, Müller S: Vegetarische und vegane Ernährung bei Kindern - Stand der Forschung und Forschungsbedarf. Forsch Komplementmed 2016;23:8188.

15 Fraser GE: The vegetarian advantage: its potential for the health of our planet, our livestock, and our neighbors! Forsch Komplementmed 2016;23:66-68.

16 United Nations: Sustainable Development Goals. 17 Goals to Transform our World. 2016. www.un.org/ sustainabledevelopment (letzter Abruf 21. März 2016). 